The results show that demand decreases with prices. They indicate that if we take a household with particular characteristics and vary only the marginal price a negative relationship holds between quantity and price. While this approach is less informative than that of section II, table 4 supports the downward sloping demand curve results rather than those produced in the Rosen framework and does not make any assumptions regarding the household's utility function.

\section{Conclusion}

This paper develops estimates of the demand for electricity in Medellin, Colombia using a method which exploits the information implicit in constrained maximization subject to a convex, but segmented linear, budget set. The estimates seem adequate statistically, fall within the accepted range of parameter estimates, and show a consistent pattern whereby richer consumers have absolutely larger price and income elasticities than do the poor. Their veracity is enhanced by similar results developed using a generalised Heckman method due to Vella (1990). This contrasts sharply with the results obtained when the standard Rosen method is applied to the same data. The formation of instruments which linearise the budget constraint was incapable with these data of identifying the downward sloping demand curve from the upward sloping supply curve.

\section{REFERENCES}

Berndt, Ernst, and Ricardo Samaniego, "Residential Electricity Demand in Mexico: A Model Distinguishing Access from Consumption," Land Economics 30 (1984), $268-277$.

Hausman, Jerry, "The Econometrics of Labor Supply on Convex Budget Sets," Economic Letters 3, (1979), $171-174$.

"The Econometrics of Nonlinear Budget Sets," Econometrica 53 (1985a) 1255-1282.

, "Taxes and Labor Supply," in A. Auerbach and M. Feldstein (eds.), Handbook of Public Economics Vol 1 (Amsterdam: North-Holland, 1985b).

Hausman, Jerry, M. Kinnucan, and D. McFadden, "A Two Level Electricity Demand Model: Evaluation of the Conneticut Time-of-Day Pricing Test," Journal of Econometrics 8 (1979), 263-289.

Heckman, James, "Sample Selection Bias as a Specification Error," Econometrica 47 (1979), 153-162.

Rosen, Harvey, "Taxes in a Labor Supply Model with Joint Wages-Hours Determination," Econometrica 44 (1976), 485-507.

Terza, Joseph, "Determinants of Household Electricity Demand: A Two Stage Probit Approach," Southern Economic Journal 52 (1986), 1131-1139.

Vella, Frank, "A Simple Estimator for Simultaneous Models with Censored Exogeneous Regressors," Monash University, Department of Econometrics, Working Paper No. 7 (1990).

Westley, Glen, The Residential and Commercial Demand for Elasticity in Paraquay, Papers on Project Analysis No. 19, Interamerican Development Bank, Washington, D.C. (1981).

, An Aggregate Time Series Study of Sectoral Electricity Demand in the Dominican Republic, Papers on Project Analysis No. 25, Interamerican Development Bank, Washington, D.C. (1984).

\title{
MULTIPLE MINIMA IN THE ESTIMATION OF MODELS WITH AUTOREGRESSIVE DISTURBANCES
}

\author{
Howard Doran and Jan Kmenta*
}

Abstract - The problem of multiple minima obtained by using the search procedure in the context of the Cochrane-Orcutt transformation disappears when the observation set is extended to include the first observation, as proposed by PraisWinsten.

\section{Introduction}

We consider, without a loss of generality, the following simple regression model with autoregressive distur-

Received for publication August 3, 1990. Revision accepted for publication April 26, 1991.

* University of New England and University of Michigan, respectively.

Kmenta's contribution was supported by the Alexandervon-Humboldt Foundation. bances:

$$
\begin{array}{ll}
Y_{t}=\alpha+\beta X_{t}+\epsilon_{t}, & t=1,2, \ldots, n, \\
\epsilon_{t}=\rho \epsilon_{t-1}+u_{t}, & |\rho|<1,
\end{array}
$$

where all the usual definitions and assumptions apply. We also assume that $u_{t}$ is normally distributed.

To remove the autoregressive $\epsilon_{t}$, one can apply the following transformation:

$$
Y_{t}^{*}=\alpha W_{t}^{*}+\beta X_{t}^{*}+u_{t}
$$

where, for $t=1$,

$$
\begin{aligned}
& Y_{t}^{*}=Y_{t} \sqrt{1-\rho^{2}}, \quad W_{t}^{*}=\sqrt{1-\rho^{2}}, \\
& X_{t}^{*}=X_{t} \sqrt{1-\rho^{2}},
\end{aligned}
$$


and, for $t=2,3, \ldots, n$,

$$
\begin{aligned}
& Y_{t}^{*}=Y_{t}-\rho Y_{t-1}, \quad W_{t}^{*}=1-\rho, \\
& X_{t}^{*}=X_{t}-\rho X_{t-1} .
\end{aligned}
$$

When the first observation, $\left(Y_{1}^{*}, W_{1}^{*}, X_{1}^{*}\right)$, is dropped, the transformation is called Cochrane-Orcutt (C-O); when it is included, the transformation is known as Prais-Winsten (P-W).

The transformed equation is usually estimated in one of two ways.

(a) Iterative procedure: Starting with the least squares estimates of the untransformed equation, the residuals are used to obtain an initial estimate of $\rho$. This estimate is used to transform the original equation and to obtain the second-stage estimates of $\alpha$ and $\beta$, and so on. The procedure is repeated until convergence.

From Huzurbazar (1948) and Oberhofer and Kmenta (1974) it follows that this procedure converges and the resulting estimator is unique and consistent regardless of whether the C-O or the P-W transformation is used.

(b) Search procedure: Suggested originally by Hildreth and Lu (1960), the sum-of-squared-errors (SSE) is computed as a function of $\rho$ and the chosen estimates $\hat{\alpha}, \hat{\beta}$ and $\hat{\rho}$ are those that correspond to minimum SSE. This minimum is located by searching over $\rho$ in the range $|\rho|<1$. It is with this method that the phenomenon of multiple minima has been documented, always in the context of the $C-O$ transformation.

\section{Multiple Minima}

The first to raise the question of multiple minima were Hildreth and Lu (1960), who provided an artificial, five-observation example of the existence of double minima of SSE. Another example, involving a more realistic model and data, was provided by Dufour et al. (1980). The issue was also more extensively treated by Oxley and Roberts (1986) who used a lagged dependent variable model. (It should be pointed out, though, that in this case the iterative $\mathrm{C}-\mathrm{O}$ estimator is inconsistent since the starting least squares estimator is inconsistent unless $\rho=0$.) A rigorous treatment of the problem of multiple minima in the context of a lagged dependent variable model can be found in Betancourt and Kelejian (1981).

In the example of Hildreth and Lu (1960) the authors found dual minima of $S S E$ at $\hat{\rho}=-0.9$ and $\hat{\rho}=0.3$, while Dufour et al. (1980) reported minima at $\hat{\rho}=0.3289$ and $\hat{\rho}=0.9318$. We have recomputed both sets of estimates using double precision and confirmed these dual minima. Thus the existence of multiple minima in small samples cannot be ruled out when the C-O transformation is used.

In this paper we examine the possibility of the existence of multiple minima when using the search procedure with the P-W transformation. To this end we
Figure 1.-SSE* FOR C-O AND P-W Estimators: Dufour ET AL. (1980) DATA

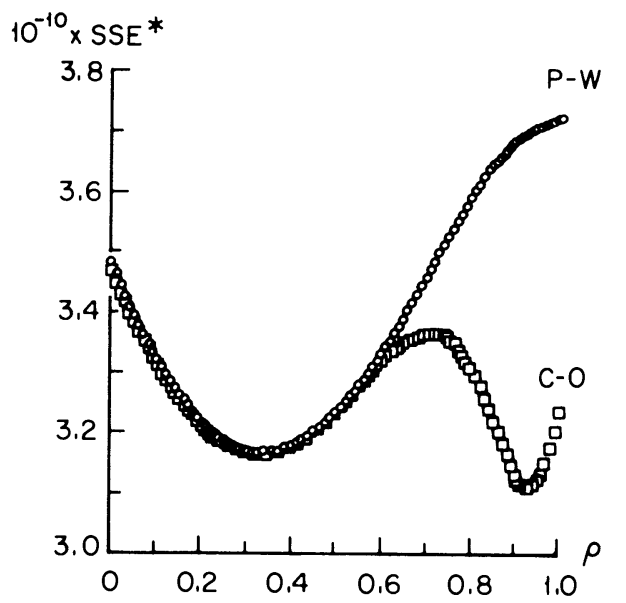

reestimated the parameters of the models of Hildreth and Lu (1960) and Dufour et al. (1980), using the authors' respective data sets but including the first observation $\left(Y_{1}^{*}, W_{1}^{*}\right.$, and $\left.X_{1}^{*}\right)$. The results turned out to be rather startling: in both cases the dual minima of $S S E$ completely disappear. The unique minimum in the Hildreth and Lu case occurs at $\hat{\rho}=-0.99$, and in the Dufour et al. case at $\hat{\rho}=0.3$. (The latter is shown in figure 1.) When using the full maximum likelihood procedure that allows for the appropriate Jacobian, the results turned out to be similar. In the case of Hildreth and $\mathrm{Lu}$, the likelihood function peaked at $\hat{\rho}=-0.78$ and in the case of Dufour et al. at $\hat{\rho}=0.315$. These unique minima correspond to the estimates of $\rho$ obtained by the iterative procedure. Since the importance of the first observation diminishes as the sample size increases, our results are consistent with the claim that the occurrence of multiple minima of the SSE curve (or multiple maxima of the likelihood function) will asymptotically disappear.

\section{An Explanation}

During our analysis of both the Hildreth and $\mathrm{Lu}$ (1960) and the Dufour et al. (1980) data sets, two features emerged. First, as emphasized above, when the transformed first observation was included, the dual minima problem disappeared. Second, when the intercept $\alpha$ was omitted from the model, the same thing happened even when the first observation was omitted. As dropping the first transformed observation converts the variable $W_{t}^{*}$ into a column of constants, there is the strong suggestion that the occurrence of dual minima is associated with the presence of a constant term in the transformed model.

To investigate the cause of the occurrence of the second, "spurious" minimum-in addition to the 
"genuine" minimum that corresponds to the maximum likelihood estimate of $\rho$-let us consider the error sum of squares $S S E^{*}$ as a function of $\rho$. This can be written as

$$
S S E^{*}=S S T^{*}\left(1-R^{* 2}\right),
$$

where $S S T^{*}$ is the total sum of squares of $Y^{*}$, and $S S E^{*}$ and $R^{* 2}$ refer to the regression of $Y^{*}$ on $W^{*}$ and $X^{*}$. We focus our attention first on the $\mathrm{C}-\mathrm{O}$ transformation, thus restricting ourselves to the observations $t=2,3, \ldots, n$ and, by implication, including an intercept in the transformed model since $W^{*}$ is constant. Note that $S S E^{*}, S S T^{*}$, and $R^{* 2}$ are all based on mean-corrected values of $X^{*}$ and $Y^{*}$.

Let us now suppose that the values of the untransformed dependent variable $Y_{t}$ in a particular sample are such that they can be adequately described by the least squares regression

$$
Y_{t}=c+d Y_{t-1}+v_{t}
$$

where, by construction, $\sum v_{t}=\sum v_{t} Y_{t-1}=0$. By "adequately described" we mean that equation (1) gives a good fit, that $Y_{t-1}$ accounts for the systematic movements in $Y_{t}$ in the sample, and that there is no systematic variation left in the sample values of $v_{t}$. It should be emphasized that equation (1) does not represent a general statement about the process of generating $Y_{t}$, which is assumed to be given by the regression model specified at the outset. With respect to equation (1), we are interested in cases where $|d|<1$.

Now, from the definition of $Y^{*}$ given as

$$
Y_{t}^{*}=Y_{t}-\rho Y_{t-1},
$$

it follows that

$$
Y_{t}^{*}=c+(d-\rho) Y_{t-1}+v_{t}
$$

and hence

$$
S S T^{*}=(d-\rho)^{2} \sum y_{t-1}^{2}+\sum v_{t}^{2}
$$

where $y_{t-1}$ is the mean-corrected value of $Y_{t-1}$. Thus $S S T^{*}$ is a quadratic function of $\rho$, having a minimum at $\rho=d$. Furthermore, the magnitude of the percentage change in $S S T^{*}$ for a small change in $\rho$ is determined by the size of $\sum v_{t}^{2}$ relative to $d^{2} \sum y_{t-1}^{2}$. If $\sum v_{t}^{2}$ is relatively small (that is, the $R^{2}$ from regression (1) is high), then a small change in $\rho$ will yield a relatively large percentage change in $S S T^{*}$, and vice versa.

Let us consider now the $\left(1-R^{* 2}\right)$ part of $S S E^{*}$ as a function of $\rho$. In the neighborhood of $\rho=d$ we have, by (2), that

$$
Y_{t}^{*} \simeq c+v_{t}
$$

and the $\mathrm{C}-\mathrm{O}$ regression of $Y_{t}^{*}$ on $W_{t}^{*}$ (a constant vector) and $X_{t}^{*}$ must yield small $R^{* 2}$ regardless of the values of $X$. In fact, for the Dufour et al. (1980) data and the C-O transformation, the values of $R^{* 2}$ corre- sponding to different values of $\rho$ are

$$
\begin{array}{lllllll}
\rho: & 0.0 & 0.1 & 0.2 & 0.3 & 0.4 & \\
R^{* 2}: & 0.76 & 0.73 & 0.68 & 0.63 & 0.55 & \\
\rho: & 0.5 & 0.6 & 0.7 & 0.8 & 0.9 & 1.0 \\
R^{* 2}: & 0.44 & 0.33 & 0.21 & 0.14 & 0.16 & 0.17
\end{array}
$$

which indicates a minimum for values of $\rho$ between 0.8 and 0.9 . When $1-R^{* 2}$ is close to 1 and insensitive (in percentage terms) to a small change in $\rho$, then the changes in $S S E^{*}$, given by the product of $S S T^{*}$ and $\left(1-R^{* 2}\right.$ ), are dominated by those of $S S T^{*}$ and hence, in the neighborhood of $\rho=d$, the product will have quadratic characteristics.

To summarize, in the cases where the descriptive least-squares regression (1) yields $|d|<1$ and the fit is reasonably good, we can expect to observe a second minimum when the $\mathrm{C}-\mathrm{O}$ transformation is used, due to the dominating influence of the quadratic $S S T^{*}$ and the minimal influence of $\left(1-R^{* 2}\right)$.

When the least-squares regression (1) was applied to the Dufour et al. (1980) data, we obtained $d=0.896$ and $R^{2}=0.74$. Thus the observed second minimum of $S S E^{*}$ at about $\rho=0.90$ (see figure 1) is to be expected. Note also that $R^{* 2}$ is at minimum very near the point where $\rho=d$.

The obvious question remaining is why, when the $\mathrm{P}-\mathrm{W}$ transformation is used (implying no intercept in the transformed model) or when the intercept $\alpha$ is dropped from the original model, a second minimum seems not to occur.

When there is no intercept in the transformed model, the preceding analysis can again be followed except that quantities are not mean-corrected and the relevant least squares regression corresponding to (1) is now

$$
Y_{t}=d_{1} Y_{t-1}+V_{t} \quad t=2,3, \ldots, n .
$$

As $d_{1}$ is simply a weighted average of the ratios $Y_{t} / Y_{t-1}$, then if in a particular sample the series $Y_{t}$ is trending upwards, we can expect $d_{1}>1$. (Note that-unlike in the case of equation (1) - it is not necessary that equation (4) gives a good fit, the only relevant point is that $d_{1}>1$.) The $\mathrm{P}-\mathrm{W}$ transformation can now be written as

$$
\begin{array}{rlrl}
Y_{t}^{*} & =\sqrt{1-\rho^{2}} Y_{t} & t & =1 \\
& =\left(d_{1}-\rho\right) Y_{t-1}+V_{t} & t & =2,3, \ldots, n
\end{array}
$$

and so

$$
S S T^{*}=\left(1-\rho^{2}\right) Y_{1}^{2}+\left(d_{1}-\rho\right)^{2} \sum Y_{t-1}^{2}+\sum V_{t}^{2}
$$

which is quadratic in $\rho$ having a minimum at

$$
\rho=K d_{1},
$$


where

$$
K=\sum_{2}^{n} Y_{t-1}^{2} / \sum_{3}^{n} Y_{t-1}^{2} .
$$

As $K \geq 1$, a spurious minimum can only occur near $\rho$ satisfying

$$
\rho \geq d_{1}>1 \text {. }
$$

Thus $S S T^{*}$, while still quadratic in $\rho$, now has a minimum outside the relevant range $|\rho|<1$. It follows that the spurious minimum which can occur when $\mathrm{C}-\mathrm{O}$ is used, will not occur when P-W is used, as long as the dependent variable $Y_{t}$ is trending upward, which is commonly the case with economic data.

Further examination of the Dufour et al. data shows that the dependent variable is trending upward, and that regression (4) yields $d_{1}=1.03$ which is outside the relevant range, as expected.

\section{Summary and Conclusion}

We believe that the reason for the occurrence of multiple minima when the $\mathrm{C}-\mathrm{O}$ search procedure is used lies in the nature of the observations on the dependent variable. Whenever a least squares regression of the form

$$
Y_{t}=c+d Y_{t-1}+v_{t}
$$

provides an adequate description of the sample data, a second minimum is likely to occur near $\rho=d$.

When the P-W transformation is used, a second minimum may still occur, but when the dependent variable is trending upward, the second minimum will be located outside the relevant region $|\rho|<1$, removing any ambiguity in the search results. Thus our results supersede the recommendation of Dufour et al. $(1980$, p. 46$)$ "to combine a search routine ... with the Cochrane-Orcutt procedure" by the recommendation always to replace the C-O transformation by the P-W transformation that requires the inclusion of the transformed first observation in the observation set.

\section{REFERENCES}

Betancourt, Roger, and Harry Kelejian, "Lagged Endogenous Variables and the Cochrane-Orcutt Procedure," Econometrica 49 (July 1981), 1073-1078.

Box, George E. P., and Gwilym M. Jenkins, Time Series Analysis (San Francisco: Holden-Day, 1976).

Dufour, Jean-Marie, Marc J. I. Gaudry, and Tran Cong Liem, "The Cochrane-Orcutt Procedure: Numerical Examples of Multiple Admissible Minima," Economics Letters 6 (1980), 43-48.

Hildreth, Clifford, and John Y. Lu, "Demand Relations with Autocorrelated Disturbances," Technical Bulletin 276, Department of Agricultural Economics, Michigan State University (1960).

Huzurbazar, V. S., "The Likelihood Equation, Consistency and the Maxima of the Likelihood Function," Annals of Eugenics 14 (1948), 185-200.

Oberhofer, Walter, and Jan Kmenta, "A General Procedure for Obtaining Maximum Likelihood Estimates in Generalized Regression Models," Econometrica (1974), 579-590.

Oxley, Leslie T., and Colin J. Roberts, “Multiple Minima and the Cochrane-Orcutt Technique: Some Initial MonteCarlo Results," Economics Letters (1986), 247-250.

\title{
THE SENSITIVITY ANALYSIS OF APPLIED GENERAL EQUILIBRIUM MODELS: COMPLETELY RANDOMIZED FACTORIAL SAMPLING DESIGNS
}

\author{
Glenn W. Harrison and H. D. Vinod*
}

\begin{abstract}
We propose a method for estimating the population mean of a distribution of solution values from applied general equilibrium models subject to parameter uncertainty. The method is illustrated by demonstrating that the "marginal excess burden" of the U.S. taxation system may be robustly bounded with a high confidence.
\end{abstract}

Received for publication April 5, 1990. Revision accepted for publication March 6, 1991.

* University of South Carolina and Fordham University, respectively.

We are grateful to Karl Scholz, Tom Rutherford, E. E. Rutström and two anonymous referees for helpful comments.

\section{Introduction}

Applied general equilibrium models have become important tools of analysis in the quantitative evaluation of trade and tax policy (see Shoven and Whalley (1984)). The solutions obtained from these models are, of course, conditional on many assumptions. One such assumption is that the set of elasticities used to calibrate the model is correct. The elasticities used are invariably obtained from "coffee table conversations" (i.e., guesses) or econometric studies. In each case there are always some uncertainties as to the true elasticity value. Estimates obtained from econometric studies are typically accompanied by standard errors 